\title{
Subcarrier Multiplexing by Chaotic Multitone Modulation
}

\author{
Cheng Juang, Shaw Tzuu Huang, Chin Yueh Liu, Wei Chung Wang, Tsung Min Hwang, Jonq Juang, and Wen Wei Lin
}

\begin{abstract}
Subcarrier multiplexing by chaotic multitone modulation is investigated. Optical chaotic light can be achieved by injecting multiple subcarriers into a self-pulsating laser diode. Synchronization between two identical chaotic systems (drive and response) can be implemented provided that the conditional Lyapunov exponents are all negative. By adding amplitude modulation (AM) signals to each subcarrier, the two systems become asymptotically synchronized. The AM signals in each subcarrier can be recovered by the introduction of a filtering process where the Lyapunov exponent of the synchronized error function matches the cutoff frequency of a first order low pass filter.
\end{abstract}

Index Terms-Asymptotic synchronization, chaotic modulation, self-pulsating laser diodes, subcarrier multiplexing.

\section{INTRODUCTION}

$\mathbf{P}$ ECORA and Carroll have shown that a chaotic system (drive system) can be synchronized with a separate chaotic system (response system) provided that the conditional Lyapunov exponents (CLE) of the drive and response systems are all negative [1]. The ability to design such synchronized systems has opened up opportunities for application of chaos to private communications. Chaotic switching, chaotic masking, and chaotic modulation are commonly used to achieve chaotic transmission [2]. Chaotic switching utilizes a parameter change in the drive system, where two chaotic states are created to bear a binary signal [3]. The important issue is that the two chaotic states are distinguishable under chaotic synchronization, and are nondistinguishable if not synchronized. For chaotic masking, a large noise-like chaotic carrier (independently-generated) is mixed with the signal at the drive end to ensure privacy [4]. At the response end, the masked signal is recovered by removing the large chaotic carrier under synchronization. In a chaotic modulation system, the chaotic oscillator in the drive end is directly modulated by the information signal. A synchronization scheme using self-pulsating laser diodes (chaotic oscillators) under a chaotic single-tone modulation has been presented [5]. Bennett et al. [6] further investigated the properties of a laser diode under directly chaotic two-tone

Manuscript received June 28, 2002; revised April 23, 2003.

C. Juang and S. T. Huang are with the Electronics Department, Ming Hsin University, Hsinchu, Taiwan, R.O.C.

C. Y. Liu and W. W. Lin are with the Department of Mathematics, National Tsing Hua University, Hsinchu, Taiwan, R.O.C.

W. C. Wang is with the Department of Mathematics, National University of Kaohsiung, Kaohsiung, Taiwan, R.O.C.

T. M. Hwang is with the Department of Mathematics, National Taiwan Normal University, Taipei, Taiwan, R.O.C.

J. Juang is with the Department of Applied Mathematics, National Chiao

Tung University, Hsinchu, Taiwan, R.O.C.

Digital Object Identifier 10.1109/JQE.2003.817662 and multitone modulation (injecting of two and $N$ sinusoidal carriers, respectively).

It is known that in broadband analog applications, one can employ a multiplexing technique where a number of baseband signals $(\Delta f)$ are amplitude modulated on a set of $N$ subcarriers having different frequencies $\left(f_{i}\right)$. These modulated subcarriers are then combined electrically to form a composite carrier that directly modulates a single optical source [7]. In this work, a possible subcarrier multiplexing based on chaotic multitone modulation is proposed, as illustrated in Fig. 1. Two identical laser diodes (with different initial conditions) are modulated by $N$ subcarriers at the transmission and receiving ends. Chaotic light output is generated under this chaotic multitone modulation so that private communication can be achieved. The two laser diodes are then synchronized according to the drive-response model. When the baseband amplitude modulation (AM) signal $(\Delta f)$ is added to each subcarrier, the two chaotic systems are no longer identical. If the AM signals are small, the two chaotic systems become asymptotically synchronized [8]. All the AM signals can be recovered by measuring the synchronized error function using a proper low-pass filter (LPF) in the receiving end. The filtering process introduces an additional Lyapunov exponent, the cutoff frequency from a first-order LPF, to the synchronized error function and may cause an increase of fractal dimension [9]. All of the AM signals can be clearly resolved if the cutoff frequency of the LPF matches well with the first Lyapunov exponent of the synchronized error function.

This paper is organized as follows. In Section II, a drive-response system model is formulated according to Pecora and Carroll's theory. In Section III, a bifurcation diagram from the Poincare map is plotted and the Lyapunov exponents for selfpulsating laser diodes under multitone chaotic modulation are calculated. Synchronization of the drive and response system are described in Section IV. Section V describes the recovery of the multiplexed signals by matching the Lyapunov exponent of the synchronized error function with the cutoff frequency of a first-order LPF.

\section{FORMULATION}

Considering a drive-response system using self-pulsating laser diodes, a drive system, described by a three-dimensional rate equation, is given by [5]

$$
\begin{aligned}
\frac{d N_{1}}{d t} & =F_{1}\left(N_{1}, N_{2}, S\right)+I+\delta(\hat{S}-S) \\
\frac{d N_{2}}{d t} & =F_{2}\left(N_{1}, N_{2}, S\right) \\
\frac{d S}{d t} & =F_{3}\left(N_{1}, N_{2}, S\right)
\end{aligned}
$$




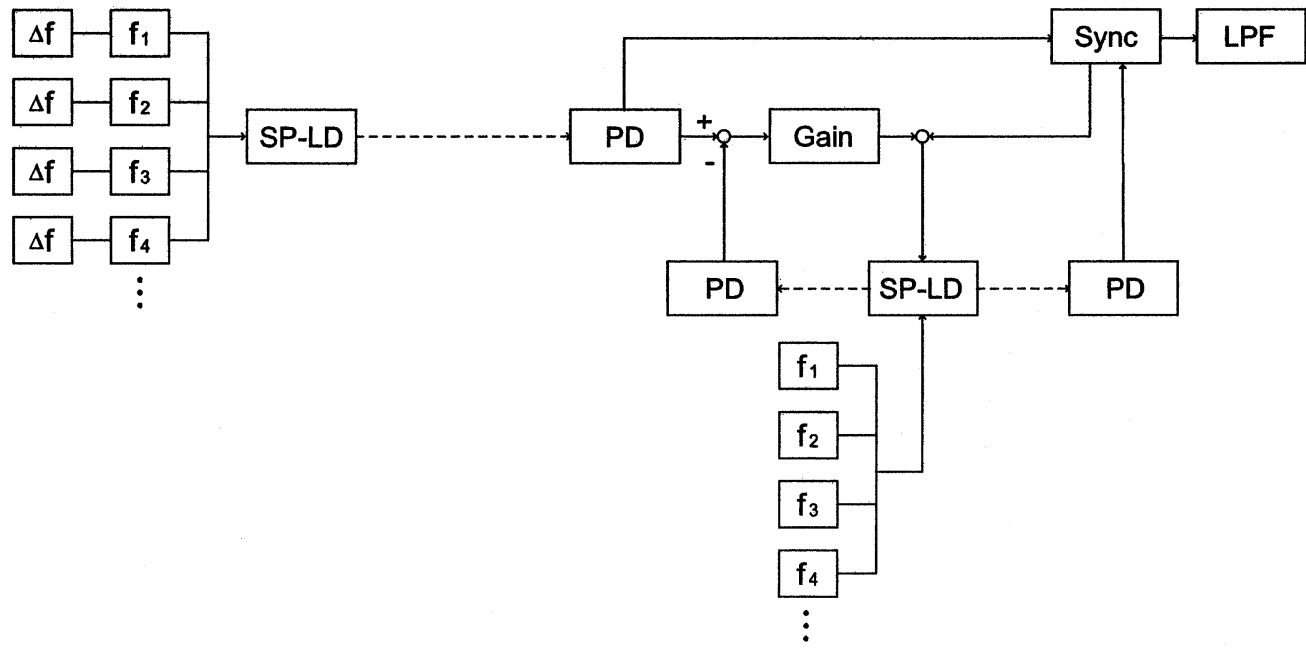

Fig. 1. Functional block diagram of subcarrier multiplexing by chaotic multitone modulation.

and the appropriate response system ( ${ }^{\circ}$ ) is given by

$$
\begin{aligned}
\frac{d \hat{N}_{1}}{d t} & =\hat{F}_{1}\left(\hat{N}_{1}, \hat{N}_{2}, \hat{S}\right)+\hat{I}+\hat{\delta}(S-\hat{S}) \\
\frac{d \hat{N}_{2}}{d t} & =\hat{F}_{2}\left(\hat{N}_{1}, \hat{N}_{2}, \hat{S}\right) \\
\frac{d \hat{S}}{d t} & =\hat{F}_{3}\left(\hat{N}_{1}, \hat{N}_{2}, \hat{S}\right)
\end{aligned}
$$

where $S$ is the photon density, $N_{1}$ is the electron density in the active region, $N_{2}$ is the electron density in the saturable absorption region, $I$ is the subcarrier current, and $\delta(\hat{S}-S)$ is the coupling term. Note that $I$ is normalized using a factor of $e V_{1}$, where $V_{1}$ is the active layer volume. The nonlinear functions $F_{1}$, $F_{2}$, and $F_{3}$ which describe the self-pulsating laser diodes can be written as [10]

$F_{1}=-\frac{k_{1} \xi_{1}}{V_{1}}\left(N_{1}-N_{g 1}\right) S-\frac{N_{1}}{\tau_{s}}-\frac{N_{1}-N_{2}}{T_{12}}$

$F_{2}=-\frac{k_{2} \xi_{2}}{V_{2}}\left(N_{2}-N_{g 2}\right) S-\frac{N_{2}}{\tau_{s}}-\frac{N_{2}-N_{1}}{T_{21}}$

$F_{3}=\left[k_{1} \xi_{1}\left(N_{1}-N_{g 1}\right)+k_{2} \xi_{2}\left(N_{2}-N_{g 2}\right)-G_{\mathrm{th}}\right] S+C \frac{N_{1} V_{1}}{\tau_{s}}$

where $\tau_{s}$ is the carrier lifetime, $\xi$ is the confinement factor, $G_{\text {th }}$ is the threshold gain level, $T$ is the carrier time diffusion constant between the two layers, $k$ is the linear approximation constant for the gain curve, $N_{g}$ is the transparent level of electron density, and $C$ is the coupling ratio between the spontaneous field and the lasing mode. The subscripts 1 and 2 describe terms in the active and absorption layers, respectively. Table I lists all the parameters of the self-pulsating laser diode obtained from [10] used in the simulation. Increasing the bias current yields a dramatic change in output light at $19.8 \mathrm{~mA}$, which corresponds to the threshold current of the laser. When above the threshold, the self-pulsating frequency $f_{0}$ increases due to the increase in
TABLE I

PARAMETERS USED FOR THE Simulation OF SELF-PUlsating LASER DiodeS

\begin{tabular}{lll}
\hline \hline parameters & value & unit \\
\hline$k_{1}$ & $3.08 \times 10^{-12}$ & $\mathrm{~m}^{3} / S$ \\
$k_{2}$ & $1.232 \times 10^{-11}$ & $\mathrm{~m}^{3} / S$ \\
$\xi_{1}$ & 0.2034 & - \\
$\xi_{2}$ & 0.1449 & - \\
$N_{g 1}$ & $1.4 \times 10^{24}$ & $m^{-3}$ \\
$N_{g 2}$ & $1.6 \times 10^{24}$ & $m^{-3}$ \\
$V_{1}$ & 72 & $\mu m^{3}$ \\
$V_{2}$ & 102.96 & $\mu m^{3}$ \\
$T_{12}$ & 2.65 & $n s$ \\
$T_{21}$ & 4.452 & $n s$ \\
$G_{t h}$ & $3.91 \times 10^{11}$ & $S^{-1}$ \\
$C$ & $1.573 \times 10^{-23}$ & $\mu m^{-3}$ \\
$\tau_{s}$ & 3 & $n s$ \\
\hline \hline
\end{tabular}

bias current a. When $\mathrm{a}=30 \mathrm{~mA}$ is injected, the corresponding $f_{0}$ is $2.28 \mathrm{GHz}$. This value is used in the calculations throughout.

\section{Synchronization of Chaotic Multitone Modulated SELF-Pulsating LASER DiODES}

By injecting $N$ sinusoidal subcarriers into a self-pulsating laser diode $\left(I=a+\sum_{i} b_{i} \sin 2 \pi f_{i} t\right)$, optical chaotic light can be obtained. In order to characterize the asymptotic behavior of the drive system, numerical computations on Poincare maps, and Lyapunov exponents are carried out for $N=4$. 


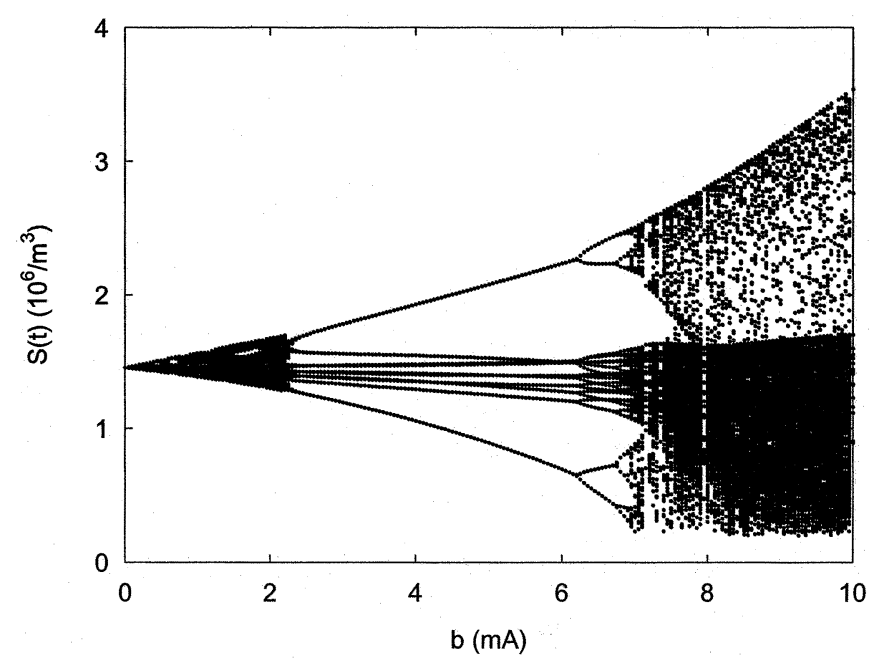

Fig. 2. Bifurcation diagram of a Poincare map with $S(t)$ versus $b$ for $N=4$, and $\mathrm{a}=30 \mathrm{~mA}$.

In the three-dimensional (3-D) phase diagram $\left(N_{1}, N_{2}\right.$, and $S$ ) of the rate equations, let $\Sigma$ be a two-dimensional (2-D) hyperplane through a point $(0,0.298,0)$ with normal direction $[0,1,0]$. The Poincare maps are taken from $\Sigma$. Fig. 2 shows the bifurcation diagram of the Poincare map with $S(t)$ versus $b=\sum_{i} b_{i}$ for $f_{i}=0.1 f_{0}, 0.2 f_{0}, 0.3 f_{0}$, and $0.4 f_{0}$. It is important to note that the four subcarrier frequencies must be chosen in a fractional ratio such that the quasi-two-period route to chaos can be maintained. When $b \in(0,2.2)$, the system has a quasi-two-period attractor. The system has a periodical window at $b \in(2.2,6.2)$ and period-doubling occurs when $b>6.2$. The phenomenon of the period-doubling routes to chaos occurs when $b \sim 6.5$.

According to Pecora and Carroll's theory, synchronization can only be achieved for all negative CLEs. The CLEs are found by calculating the Lyapunov exponents for the entire system and comparing these to the Lyapunov exponents of the drive system. The remaining Lyapunov exponents are the CLEs. A simple method is to construct a difference system: let $E_{N_{1}}=$ $N_{1}-\hat{N}_{1}, E_{N_{2}}=N_{2}-\hat{N}_{2}$, and $E_{S}=S-\hat{S}$. The difference system becomes

$$
\frac{d E(t)}{d t}=A E(t)
$$

The real part of the eigenvalues $\left(\lambda_{1}, \lambda_{2}\right.$, and $\left.\lambda_{3}\right)$ of $A^{-1}$ are the CLEs by definition. If all the CLEs are negative $\lim _{t \rightarrow \infty} E(t)=0$, the two systems will be synchronized. If there is a positive CLE, the difference system will grow further apart as $t \rightarrow \infty$. Thus, by using a positive CLE, synchronization can be established between the drive and response system under chaotic multitone modulation.

\section{ReCOVERY OF MultiPleXed SignAls}

AM signals are added to each subcarrier and the resulting injection current is $I=a+\sum_{i}\left(b_{i}+\Delta b \sin 2 \pi \Delta f t\right) \sin 2 \pi f_{i} t$, where $\Delta f$ is the AM signal frequency, and $\Delta b$ is the AM signal amplitude. In the response system, $\hat{b}_{i}$ remains a constant current. Thus, the two chaotic systems are no longer identical. If $\Delta b$ is

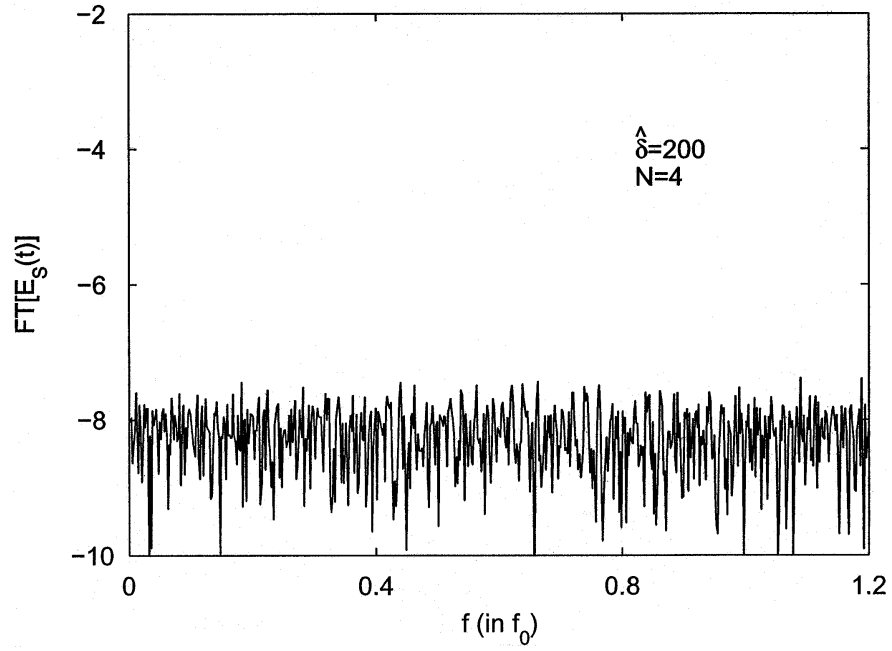

Fig. 3. Frequency spectrum of the four subcarrier synchronization error function before applying the filter.

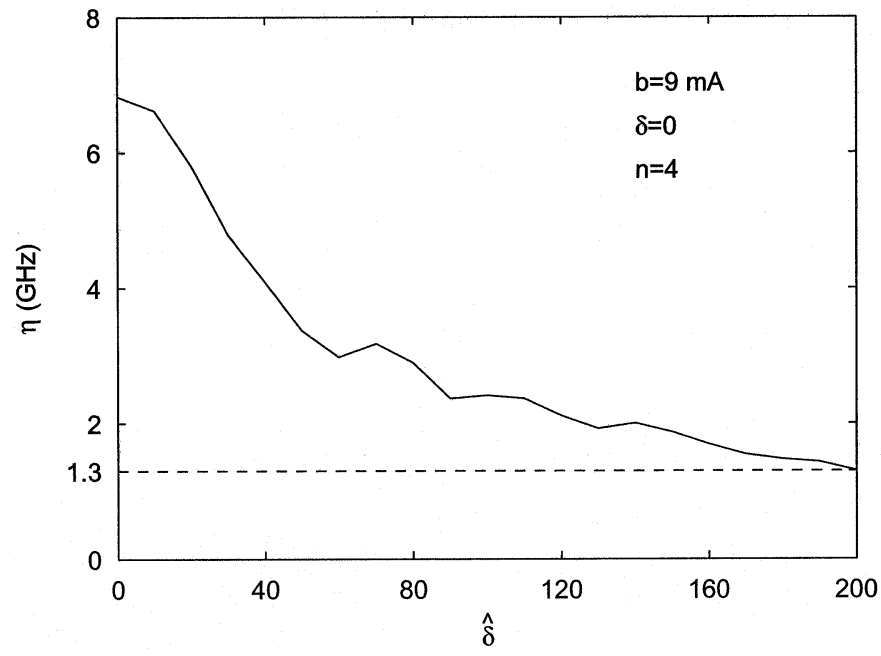

Fig. 4. First Lyapunov exponent of the synchronized error function versus $\hat{\delta}$ for $\delta=0, N=4, \mathrm{~b}=9 \mathrm{~mA}$, and $\mathrm{a}=30 \mathrm{~mA}$.

small, the two chaotic systems become asymptotically synchronized. The synchronized error function $E_{S}(t)$ can be described by [8]

$$
\lim _{t \rightarrow \infty}\left|E_{S}(t)\right|=\lim _{t \rightarrow \infty}|S(t)-\hat{S}(t)|=k\left[\frac{|\epsilon|}{(\delta+\hat{\delta})}\right]
$$

where $k$ is the proportional constant and $\epsilon$, the difference term between the drive and response systems, which is given by

$$
|\epsilon|=\left|\sum_{i} \Delta b \sin 2 \pi \Delta f t \sin 2 \pi f_{i} t\right| .
$$

Fig. 3 shows the first Lyapunov exponent $\gamma_{1}$ of the synchronized error function versus $\hat{\delta}$ for $\delta=0, N=4, \mathrm{~b}=9 \mathrm{~mA}$, and $a=30 \mathrm{~mA}$. The positive first Lyapunov exponent implies the chaotic behaviors of the synchronized error function. Fig. 3 shows the frequency spectrum of the synchronization error function, which has a complex and unpredictable pattern. The signal frequency $\Delta f$ is well hidden by the chaotic modulation. Thus, the purposes of the private communication can be achieved. 


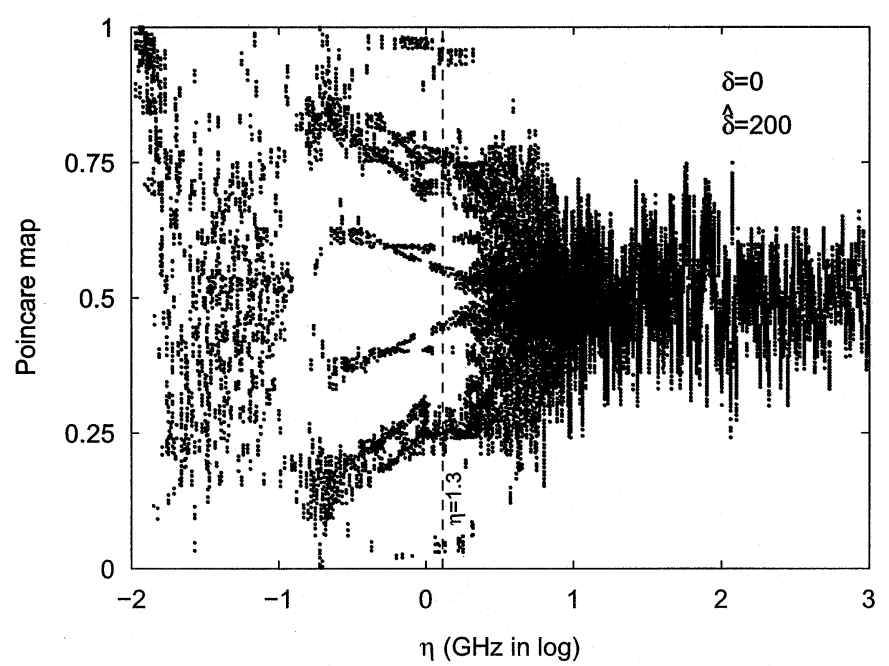

Fig. 5. Poincare cross section of $X(t)$ versus $X(t-\tau)$ against the cutoff frequency $\eta$ for $\hat{\delta}=200, \delta=0, N=4, \mathrm{~b}=9 \mathrm{~mA}$, and a $=30 \mathrm{~mA}$, where $\tau=\left(10 f_{0}\right)^{-1}$.

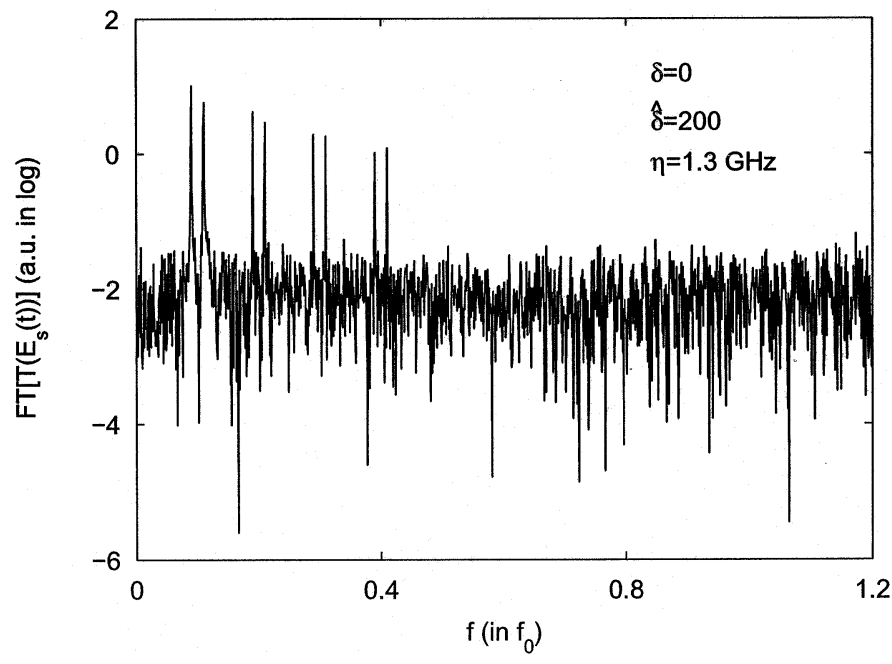

Fig. 6. Frequency spectrum of the output of the LPF $X(t)$ for $\eta=1.3 \mathrm{GHz}$, $\hat{\delta}=200, \delta=0, N=4, \mathrm{~b}=9 \mathrm{~mA}$, and $\mathrm{a}=30 \mathrm{~mA}$.

The introduction of a linear LPF to the original differential model can be described by [9]

$$
\dot{X}(t)=-\eta X(t)+E_{S}(t)
$$

where $X(t)$ is the output of the filter and $\eta$ is the cutoff frequency. Fig. 5 plots the Poincare cross section of the $X(t)$ versus $X(t-\tau)$ against the cutoff frequency $\eta$ for $\hat{\delta}=200$, $\delta=0, N=4, \mathrm{~b}=9 \mathrm{~mA}$, and $\mathrm{a}=30 \mathrm{~mA}$, where $\tau$ is a delay time chosen to be $\left(10 f_{0}\right)^{-1}$. Note that for $\hat{\delta}=200$, the first Lyapunov exponent of the synchronized error function is $1.3 \mathrm{GHz}$ from Fig. 3. For a large cutoff frequency, contribution of the differential term can be ignored $\left(E_{S}(t) \sim X(t)\right)$. Thus, the Poincare cross section $(\eta>3 \mathrm{GHz})$ shows a continuous dense attractor-a typical behavior of a chaotic waveform. For a very small cutoff frequency, $E_{S}(t) \sim \dot{X}(t)$. The Poincare cross section $(\eta<0.2 \mathrm{GHz})$ shows a broken attractor-an integration effect of the dense attractor. However, when the

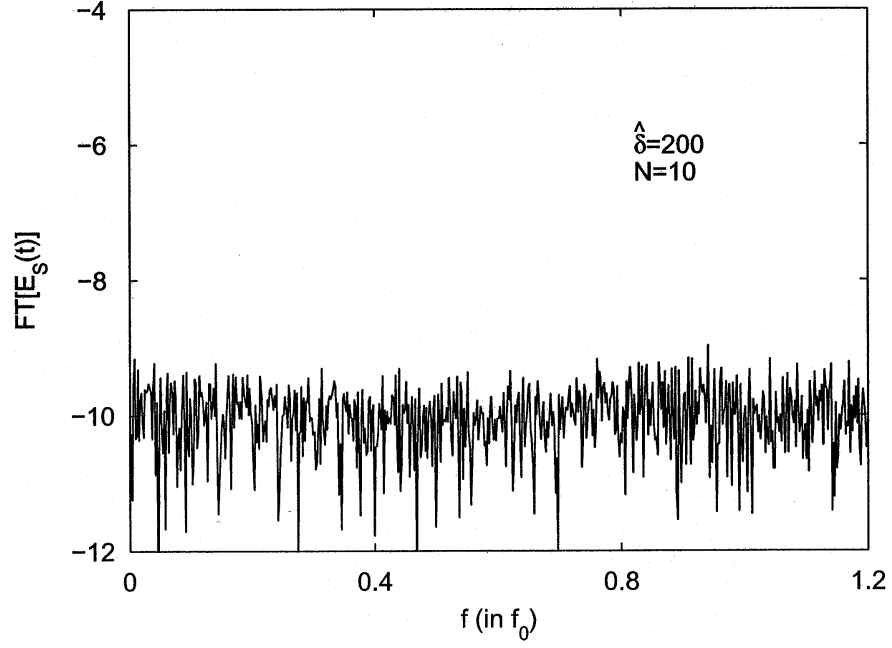

Fig. 7. Frequency spectrum of the ten subcarrier synchronization error function before applying the filter.

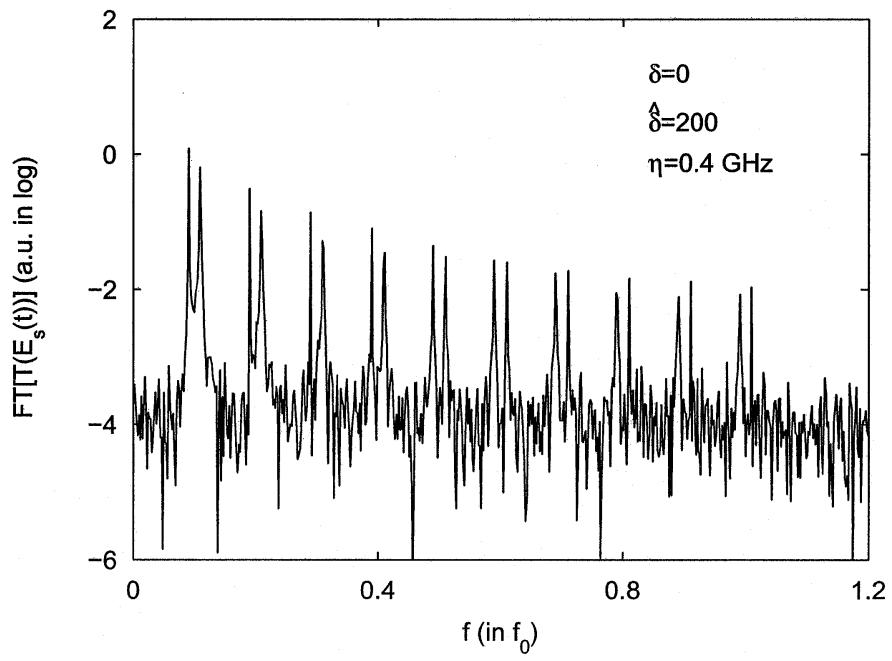

Fig. 8. Frequency spectrum of the output of the LPF $X(t)$ for $\eta=0.4 \mathrm{GHz}$, $\hat{\delta}=200, \delta=0, N=10, \mathrm{~b}=7 \mathrm{~mA}$, and $\mathrm{a}=30 \mathrm{~mA}$.

cutoff frequency matches the first Lyapunov exponent of the synchronized error function $(\eta \sim 1.3 \mathrm{GHz})$, as suggested in [9], an increase in fractal dimensional occurs. Therefore, the dense attractors become periodic and the embedded signals can be recovered.

The recovery of the multiplexed signals can also be verified in the frequency domain. $\Delta b \sin 2 \pi \Delta f t \sin 2 \pi f_{i} t$ gives the sum frequency $\left(f_{i}+\Delta f\right)$ and the difference frequency $\left(f_{i}-\Delta f\right)$ at each subcarrier. Fig. 6 shows the frequency spectrum of the output of the LPF $X(t)$ for $\eta=1.3 \mathrm{GHz}, \hat{\delta}=200, \delta=0$, $N=4, b=9 \mathrm{~mA}$, and a $=30 \mathrm{~mA}$. Two distinct signal peaks are found at $\left(f_{i}+\Delta f\right)$ and $\left(f_{i}-\Delta f\right)$ in each subcarrier. Thus, the subcarrier multiplexing for $N=4$ can be established.

\section{COMPARISON of $N=4$ AND $N=10$ MultipleXed SIGNALS}

To further test the validity of this filtering process, a ten subcarrier system $\left(f_{i}=0.1 \times i \times f_{0}, \mathrm{i}=1 \ldots 10\right)$ is constructed. Fig. 7 shows the frequency spectrum of the synchronization 


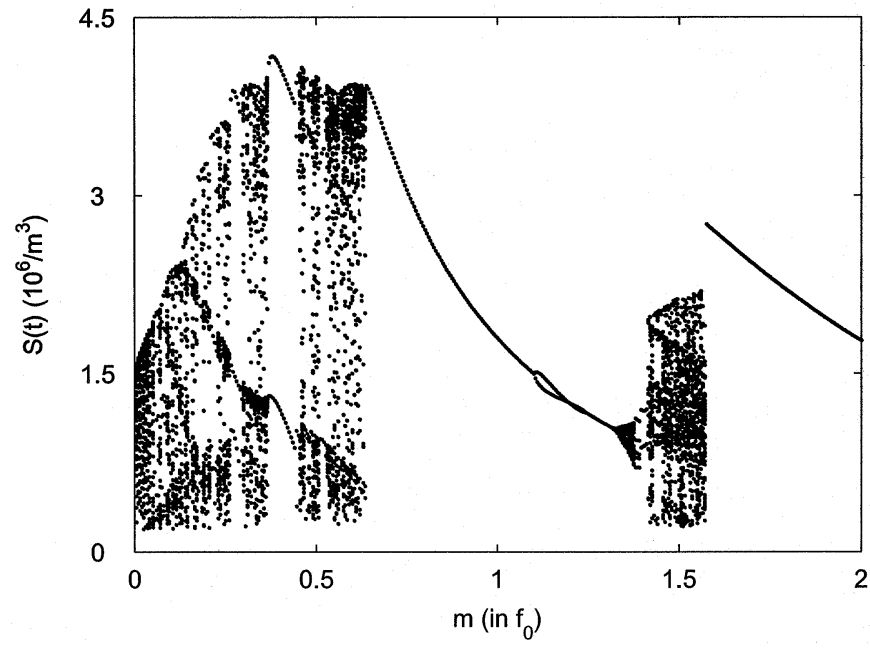

Fig. 9. Bifurcation diagram of $S(t)$ versus $m$. $N=1$ corresponds to a frequency modulation index of 0.1 .

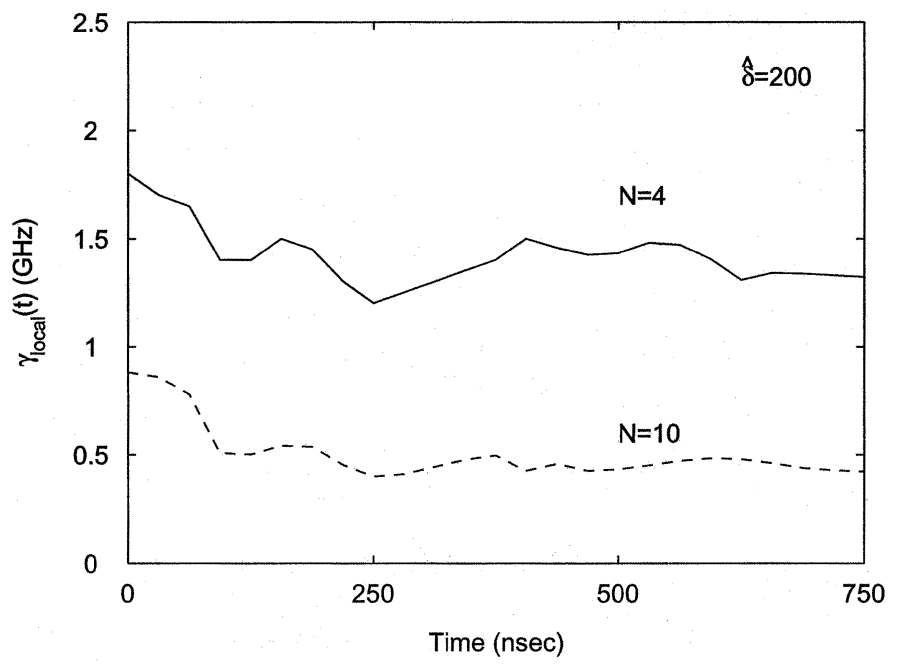

Fig. 10. Comparison of the local Lyapunov exponent for $N=4$ and $N=10$.

error function before applying the filter. Similarly, the spectrum has a complex and unpredictable pattern and the signal frequency $\Delta f$ is well hidden.

In this system, the first Lyapunov exponent of the synchronized error function is $0.4 \mathrm{GHz}$. Therefore, the cutoff frequency of LPF is chosen to be $0.4 \mathrm{GHz}$ accordingly. Fig. 8 shows the frequency spectrum of the output of the LPF $X(t)$ for $\eta=$ $0.4 \mathrm{GHz}, \hat{\delta}=200, \delta=0, N=10, \mathrm{~b}=7 \mathrm{~mA}$, and a $=30 \mathrm{~mA}$. Similarly, two distinct signal peaks are found at $\left(f_{i}+\Delta f\right)$ and $\left(f_{i}-\Delta f\right)$ in each subcarrier. Thus, the subcarrier multiplexing for $N=10$ can also be established.

When taking into consideration of each subcarrier frequency, it is difficult to place all the subcarrier in the chaotic regions. The chaotic orbit can be destroyed while the subcarrier frequency is approaching to the natural frequency $f_{0}[11]$. In this case, $N=1 \ldots 6$ exhibit chaotic orbit while $N=7 \ldots 10$ show periodic window, as shown in Fig. 9, where $m$ is the frequency modulation index. $\mathrm{N}=1$ corresponds to a frequency modulation index of 0.1 . Thus, the summing effects of the ten subcarrier system shows less "chaos" than the four subcarrier system. It is also evident by plotting the local Lyapunov exponent (in Fig. 10). The ten subcarrier system has a smaller local Lyapunov exponent all the way.

\section{CONCLUSIONS}

In conclusion, self-pulsating laser diodes can be used as a chaotic multitone modulation device to change sinusoidal electronic signals into optical chaotic light. Drive and response systems become asymptotically synchronized when AM signals are added to each subcarrier in the drive system. The AM signals in each subcarrier can be recovered by matching the Lyapunov exponent of the synchronized error function with the cutoff frequency of a first-order LPF. The numerical results indicate four and ten subcarrier multiplexing systems can be established.

\section{REFERENCES}

[1] L. M. Pecore and T. L. Carroll, "Synchronization in chaotic systems," Phys. Rev. Lett., vol. 64, pp. 821-824, 1990.

[2] M. Delgado-Restituto, A. Rodriguez-Vazquez, R. Lopez-Ahumada, and M. Linan, "Chaotic synchronization using monolithic Chua oscillators," Int. J. Electron., vol. 79, pp. 775-785, 1995.

[3] K. M. Cuomo, A. V. Oppenheim, and S. H. Strogatz, "Synchronization of Lorenz-based chaotic circuits with applications to communication," IEEE Trans. Circuit Syst. II, vol. 40, pp. 626-633, 1993.

[4] H. D. I. Abarbanel, M. B. Kennel, L. Illing, S. Tang, H. F. Chen, and J. M. Liu, "Synchronization and communication using semiconductor lasers with optoelectronic feedback," IEEE J. Quantum Electron., vol. 37, pp. 1301-1311, 2001.

[5] C. Juang, T. M. Hwang, J. Juang, and W. W. Lin, "A synchronization scheme using self-pulsating laser diodes in optical chaotic communication," IEEE J. Quantum Electron., vol. 36, pp. 300-304, 2000.

[6] S. Bennett, C. M. Snowden, and S. Iezekiel, "Nonlinear dynamics in directly modulated multiple-quantum-well laser diodes," IEEE J. Quantum Electron., vol. 33, pp. 2076-2083, 1997.

[7] C. S. Ih and W. Gu, "Fiber induced distortions in subcarrier multiplexed lightwave systems," IEEE J. Select. Areas Commun., vol. 8, pp. 1296-1303, 1990

[8] C. Juang, T. M. Hwang, J. Juang, and W. W. Lin, "Optical chaotic AM demodulation by asymptotic synchronization," IEEE Photon. Technol. Lett., vol. 12, pp. 179-181, 2000.

[9] R. Badii, G. Broggi, B. Derighetti, M. Ravani, S. Ciliberto, A. Poloti, and M. A. Rubio, "Dimension increase in filtered chaotic signals," Phys. Rev. Lett., vol. 60, pp. 979-982, 1988.

[10] M. Yamada, "A theoretical analysis of self-sustained pulsation phenomena in narrow-strip semiconductor lasers," IEEE J. Quantum Electron., vol. 29, pp. 1330-1336, 1993.

[11] C. Juang, M. R. Chen, and J. Juang, "Nonlinear dynamics of self-pulsating laser diodes under external drive," Opt. Lett., vol. 24, pp. 1346-1348, 1999

Cheng Juang received the Ph.D. degree in electrical engineering from the University of Washington, Seattle, in 1990.

Currently, he is a Full Professor in the Electronics Department, Ming Hsin University, Hsinchu, Taiwan, R.O.C.

Shaw-Tzuu Huang received the M.S. degree in electrical engineering from Tatung University, Taiwan, R.O.C., in 1984.

Currently, he is a Lecturer in the Electronics Department, Ming Hsin University, Hsinchu, Taiwan, R.O.C.

Chin-Yueh Liu received the M.S. degree in applied mathematics from National Tsing Hua University, Hsinchu, Taiwan, R.O.C., in 2000.

Currently, he is a Research Assistant in the National Center of Theoretical Science, Hsinchu, Taiwan, R.O.C. 
Weichung Wang received the Ph.D. degree in applied mathematics from the University of Maryland, College Park, in 1996.

$\mathrm{He}$ is currently an Associate Professor in the Department of Applied Mathematics, National University of Kaohsiung, Taiwan, R.O.C.

Tsung-Min Hwang received the Ph.D. degree in applied mathematics from National Tsing Hua University, Hsinchu, Taiwan, R.O.C., in 1994.

Currently, he is an Associate Professor in the Department of Mathematics, Taiwan Normal University, Taipei, Taiwan, R.O.C.
Jonq Juang received the the Ph.D. degree in mathematics from Texas Tech University, Lubbock, in 1988.

Currently, he is a Full Professor in the Department of Applied Mathematics, National Chiao Tung University, Hsinchu, Taiwan, R.O.C.

Wen-Wei Lin received the Ph.D. degree from Bielefeld University, Germany, in 1985.

Currently, he is a Full Professor in the Department of Mathematics, National Tsing Hua University, Hsinchu, Taiwan, R.O.C. 